\title{
Editorial
}

\section{Mathematical Methods Applied to Digital Image Processing}

\author{
Yi-Hung Liu, ${ }^{1}$ Chung-Hao Chen, ${ }^{2}$ and Paul C.-P. Chao ${ }^{3}$ \\ ${ }^{1}$ Department of Mechanical Engineering, Chung Yuan Christian University, Chungli 32023, Taiwan \\ ${ }^{2}$ Department of Electrical and Computer Engineering, Old Dominion University, Norfolk, VA 23529, USA \\ ${ }^{3}$ Department of Electrical Engineering, National Chiao Tung University, Hsinchu 30010, Taiwan \\ Correspondence should be addressed to Yi-Hung Liu; lyh@cycu.edu.tw
}

Received 5 March 2014; Accepted 5 March 2014; Published 22 April 2014

Copyright (c) 2014 Yi-Hung Liu et al. This is an open access article distributed under the Creative Commons Attribution License, which permits unrestricted use, distribution, and reproduction in any medium, provided the original work is properly cited.

\section{Introduction}

Digital image processing (DIP) is an important research area since it spans a variety of applications. Although over the past few decades there has been a rapid rise in this field, there still remain issues to address. Examples include image coding, image restoration, 3D image processing, feature extraction and analysis, moving object detection, and face recognition. To deal with these issues, the use of sophisticated and robust mathematical algorithms plays a crucial role. The aim of this special issue is to provide an opportunity for researchers to publish their latest theoretical and technological achievements in mathematical methods and their various applications related to DIP. This special issue covers topics related to the development of mathematical methods and their applications. It has a total of twenty-four highquality papers covering various important topics in DIP, including image preprocessing, image encoding/decoding, stereo image reconstruction, dimensionality and data size reduction, and applications.

\section{Image Preprocessing}

One of the topics covered in this special issue is related to the image preprocessing methods, including segmentation, thresholding, denoising, image interpolation, calibration of cameras, and image restoration. There are twelve papers related to this topic, occupying half of the papers included in this special issue. The paper entitled "An improved adaptive deconvolution algorithm for single image deblurring" by $\mathrm{H}$.C. Tsai and J.-L. Wu proposes an adaptive regularization method, which can preserve image details and suppress ringing artifacts simultaneously. Their results carried out on synthesized and real images show that their method can restore latent image with much fewer ringing and favors the sharp edges. S. Pan et al. propose the paper entitled "Optimal $O(1)$ bilateral filter with arbitrary spatial and range kernels using sparse approximation." In this paper, an approximating arbitrary spatial kernel using a fixed number of boxes is presented. The multiple-box spatial kernel can be applied in many $O(1)$ acceleration schemes in addition to the histogrambased one. Their results show that the proposed method has better accuracy in approximating the bilateral filter with Gaussian spatial kernel, compared with previous histogrambased methods.

Then, the paper titled "Fast total-variation image deconvolution with adaptive parameter estimation via split Bregman method" by $\mathrm{C}$. He et al. studies how to balance the totalvariation (TV) regularization and data fidelity, which has considered a challenging issue in image restoration. Their proposed method extends the classical split Bregman method to a new fast algorithm, which simultaneously estimates the regularization parameter and restores the blurred image. The regularization parameter is refreshed conveniently in a closed form according to Morozov's discrepancy principle. Another paper of this topic is "Robust calibration of cameras with telephoto lens using regularized least squares" by M. Liang et al. This paper has two main contributions. First, they present a first-order error analysis that shows the relation between focal length and estimation uncertainties of camera parameters. To our knowledge, this error analysis with respect to focal length has not been studied in the area of camera calibration. 
Second, they propose a robust algorithm to calibrate the camera with a long focal length without using additional devices: the covariance of camera parameters can be reduced greatly.

The next paper of this topic is "A block-based regularized approach for image interpolation" by L. Chen et al. This paper studies how to render a high-resolution image from a lowresolution image. Classical interpolation techniques estimate the missing pixels from the surrounding pixels based on a pixel-by-pixel basis. In contrast, this paper presents a new efficient algorithm for image interpolation based on regularization theory, which formulates the interpolation problem as an optimization problem in which the cost function consists of a data fidelity term and regularization functional. The computational cost of this algorithm is further reduced by incorporating Kronecker product and singular value decomposition. The effect of regularization on the interpolation results is analyzed in this paper. Next, the paper entitled "Sensor selection and integration to improve video segmentation in complex environments" coauthored by A. R. Reckley et al. proposes a technique to integrate spatiotemporal signatures of an object of interest from different sensing modalities into a video segmentation method in order to improve object detection and tracking in dynamic, complex scenes. Their proposed algorithm utilizes the dynamic interaction information between the object of interest and background to differentiate between mistakenly segmented components and the desired component. S. Tehsin et al. proposed the paper entitled "Fuzzy-based segmentation for variable font-sized text extraction from images/videos," which addresses the issue of text localization and detection by using a novel fuzzy-based segmentation method.

Moreover, Poissonian image deconvolution is a key issue in various applications, such as astronomical imaging, medical imaging, and electronic microscope imaging. To address this issue, Y. Shi et al. propose the paper "Poissonian image deconvolution via sparse and redundant representations and framelet regularization". In this paper, an approach that combines analysis with synthesis methods is proposed. The aim is to address the Poissonian image deconvolution problem by minimizing an energy functional. The minimization problem can be efficiently solved by the split Bregman technique. D.-X. $\mathrm{Xia}$ et al. propose the paper entitled "Fast threshold selection algorithm of infrared human images based on two-dimensional fuzzy Tsallis entropy." In this paper, a fast thresholding method of infrared human images based on two-dimensional fuzzy Tsallis entropy is introduced. First, to address the fuzziness of infrared image, the fuzzy Tsallis entropy of objects and that of background are defined, respectively, according to probability partition principle. Next, this newly defined entropy is extended to two dimensions to make good use of spatial information to deal with the noise in infrared images, and correspondingly a fast computation method of two-dimensional fuzzy Tsallis entropy is put forward to reduce its computation complexity.

Another paper to this set is "Image denoising using total variation model guided by steerable filter" by W. Zhang et al. This paper proposes an adaptive total variation (TV) model by introducing the steerable filter into the TV-based diffusion process for image filtering. The proposed model can provide a better image processing tool which enables noise removal, edge-preserving, and staircase suppression. The paper also that belongs to this topic is the one entitled " $A$ global multilevel thresholding using differential evolution approach" by K. Charansiriphaisan et al. The optimal Otsu threshold values of multilevel image thresholding are necessary for some applications and a global search algorithm is required. Previously, differential evolution (DE) algorithm has been used successfully for solving this problem. However, the difficulty of problem grows exponentially when the number of thresholds increases. To overcome this difficulty, an improved DE using new mutation strategy is proposed in this paper. The last paper of this topic is related to image restoration. W. Qidi, et al. propose the paper entitled "The nonlocal sparse reconstruction algorithm by similarity measurement with Shearlet feature vector," which supplies a nonlocal sparsity reconstruction algorithm with similarity measurement to improve the accuracy of conventional methods of image restoration. To improve the performance of restoration results, this paper proposes two schemes to dictionary learning and sparse coding, respectively. In the part of the dictionary learning, the similarity between patches from degraded image by constructing the Shearlet feature vector is measured. In the part of sparse coding, an optimal objective function with the coding residual item is performed, which can suppress the residual between the estimate coding and true sparse coding. The derivation of self-adaptive regularization parameter in optimization under the Bayesian framework is also provided, which can make the performance better.

\section{Stereo Image Reconstruction}

In this special issue, there are three papers related to the topic of stereo image reconstruction. The homography between image pairs is normally estimated by minimizing a suitable cost function, given a set of 2D keypoint correspondences. The correspondences are typically established using descriptor distance of keypoints. However, the correspondences are often incorrect due to ambiguous descriptors which can introduce errors into following homography computing step. There have been numerous attempts to filter out these erroneous correspondences, but it is unlikely to always achieve perfect matching. To deal with this problem, W. Mou et al. propose the paper entitled "Robust homography estimation based on nonlinear least squares optimization," in which a nonlinear least squares optimization approach is presented to compute homography such that false matches have no or little effect on computed homography.

Moreover, developing matching algorithms from stereo image pairs to obtain correct disparity maps for $3 \mathrm{D}$ reconstruction has also been the focus of intensive research. A constant computational complexity algorithm to calculate dissimilarity aggregation in assessing disparity based on separable successive weighted summation (SWS) among horizontal and vertical directions was proposed but still not satisfactory. To address this issue, C.-T. Zhu et al. propose the paper entitled "Efficient stereo matching with decoupled 
dissimilarity measure using successive weighted summation," which presents a novel method enabling decoupled dissimilarity measure in the aggregation, further improving the accuracy and robustness of stereo correspondence. The aggregated cost is also used to refine disparities based on a local curve-fitting procedure.

Then, the paper entitled "Rendering distortion estimation model for 3D high efficiency depth coding" by Q. Zhang et al. proposes a modified distortion estimation model based on view rendering distortion. This model can be applied to the high efficiency video coding (HEVC) rate distortion cost function process for rendering view quality optimization. Their experimental results on various $3 \mathrm{D}$ video sequences show that this model can provide about $31 \% \mathrm{BD}$-rate savings in comparison with HEVC simulcast and $1.3 \mathrm{~dB}$ BD-PSNR coding gain for the rendered view.

\section{Image Encoding and Decoding}

Two papers published in this special issue are focused on image encoding and decoding. The paper entitled " $2 D$ barcode image decoding" coauthored by J.-A. Lin and C.$\mathrm{S}$. Fuh studies how to decode various quick response (QR) code images efficiently and accurately. This paper revises the traditional decoding procedure by proposing a serial of carefully designed preprocessing methods. The decoding procedure consists of image binarization, QR code extraction, perspective transformation and resampling, and error correction. By these steps, different types of QR code images can be recognized accurately. K. T. Lin and S. L. Yeh propose the paper entitled "Hiding a covert digital image by assembling the RSA encryption method and the binary encoding method," which integrates the Rivest-Shamir-Adleman encryption method and the binary encoding method to form a hybrid hiding method that can hide a covert digital image into a dot-matrix holographic image. The pixels of the dotmatrix holographic image contain seven groups of codes used for reconstructing the covert image, and the reconstructed covert image derived from the dot-matrix holographic image and the original covert image are exactly the same.

\section{Dimensionality and Dataset Size Reduction}

There are two papers in this topic. In image recognition, feature extraction plays a critical role. Feature extraction typically suffers from the problem of high dimensionality. Therefore, the issue of dimension reduction is of primary importance. The convex nonnegative matrix factorization (CNMF) is a variation of nonnegative matrix factorization (NMF) in which each cluster is expressed by a linear combination of the data points and each data point is represented by a linear combination of the cluster centers. When there exists nonlinearity in the manifold structure, both NMF and CNMF are incapable of characterizing the geometric structure of the data. The paper "Neighborhood preserving convex nonnegative matrix factorization" by J. Wei et al. introduces a neighborhood preserving convex nonnegative matrix factorization (NPCNMF), which imposes an additional constraint on CNMF that each data point can be represented as a linear combination of its neighbors. This method is able to reap the benefits of both nonnegative data factorization and the purpose of manifold structure. The feasibility and effectiveness of NPCNMF are verified on several standard data sets with promising results.

Another major problem of pattern recognition systems is due to the large volume of training datasets including duplicate and similar training samples. As a result, a large memory is required for saving data, as well as the high time complexity for training the algorithms. The paper entitled "A new dataset size reduction approach for PCA-based classification in OCR application" by M. A. Shayegan and S. Aghabozorgi reports on the use of a version of the Modified Frequency Diagram technique for dataset size reduction. In this new proposed technique, a big training dataset is rearranged and then sieved. The sieved training dataset along with automatic feature extraction/selection operation using Principal Component Analysis (PCA) is used in an optical character recognition (OCR) application. The experimental results obtained when using the proposed system on one of the biggest handwritten Arabic/Farsi numeral standard OCR datasets, Hoda, show about $97 \%$ accuracy in the recognition rate. The recognition speed increased by 2.28 times, while the accuracy decreased only by $0.7 \%$.

\section{Applications}

In this special issue, five papers are related to DIP applications. Among the five papers, three are directly related to face detection and recognition. Y.-H. Liu et al. propose the paper entitled "A support vector data description committee for face detection," which develops a one-class learning-based face detector called support vector data description (SVDD) committee, which consists of several SVDD members, each of which is trained on a subset of face patterns. Since nonfaces are not required in the training of the SVDD committee, face detection accuracy of SVDD committee is independent of the nonface training patterns. Experiments carried out on the extended MIT face dataset show that the proposed SVDD committee can achieve better face detection accuracy than the widely used SVM face detector. Z. Jian et al. propose the paper entitled "A fast iterative pursuit algorithm in robust face recognition based on sparse representation." In this paper, a relatively fast pursuit algorithm for face recognition is proposed. The recognition rates on ORL database, Yale database, and FERET database are $95.5 \%, 93.87 \%$, and $92.29 \%$, respectively. The recognition performance under various levels of occlusion and corruption is also experimentally proved to be significantly enhanced. The paper "An improved metric learning approach for degraded face recognition" by G. Zou et al. also addresses the face recognition problem. To solve the matching problem of the elements in different data collections, an improved coupled metric learning approach is proposed in this paper. Experiments based on Yale and CAS-PEAL-R1 face databases demonstrate that the proposed kernel coupled approach performs better in low-resolution 
and fuzzy face recognition and can reduce the computing time.

In addition to face recognition, other problems are also challenging in real-word applications. J. Xiang et al. propose the paper entitled "Moving object detection and shadow removing under changing illumination condition" that addresses the problem of moving object segmentation by proposing a local intensity ratio model that is robust to illumination change. Y. H. Liu et al. propose the paper entitled "Low-resolution tactile image recognition for automated robotic assembly using kernel PCA-based feature fusion and multiple kernel learning-based support vector machine." In this paper, a robust tactile sensing image recognition scheme is proposed, a kernel PCA feature fusion layer is performed to extract distinguishing features from a set of candidate features including Fourier descriptors and geometric features, and a multiple kernel learning support vector machine is used as the classifier. The proposed recognition scheme is able to achieve a high recognition rate of over $85 \%$ for the classification of 12 commonly used metal parts in industrial applications.

\section{Acknowledgments}

The guest editors would like to thank the authors and reviewers of the journal who worked on the publication of this special issue.

Yi-Hung Liu

Chung-Hao Chen

Paul C.-P. Chao 


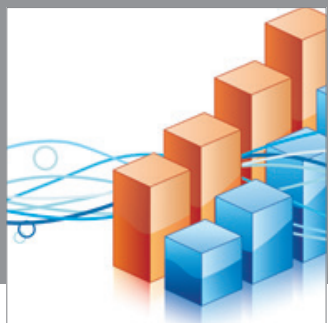

Advances in

Operations Research

mansans

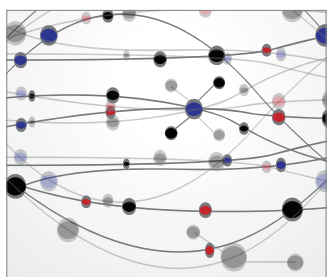

The Scientific World Journal
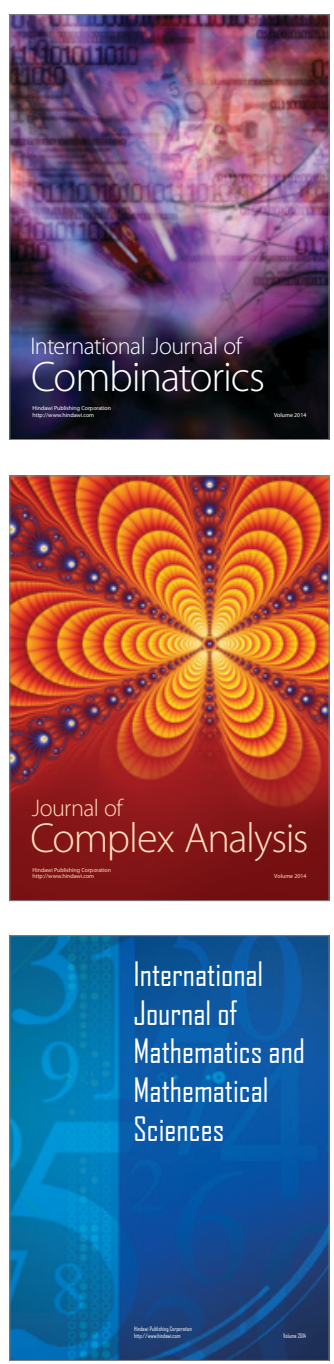
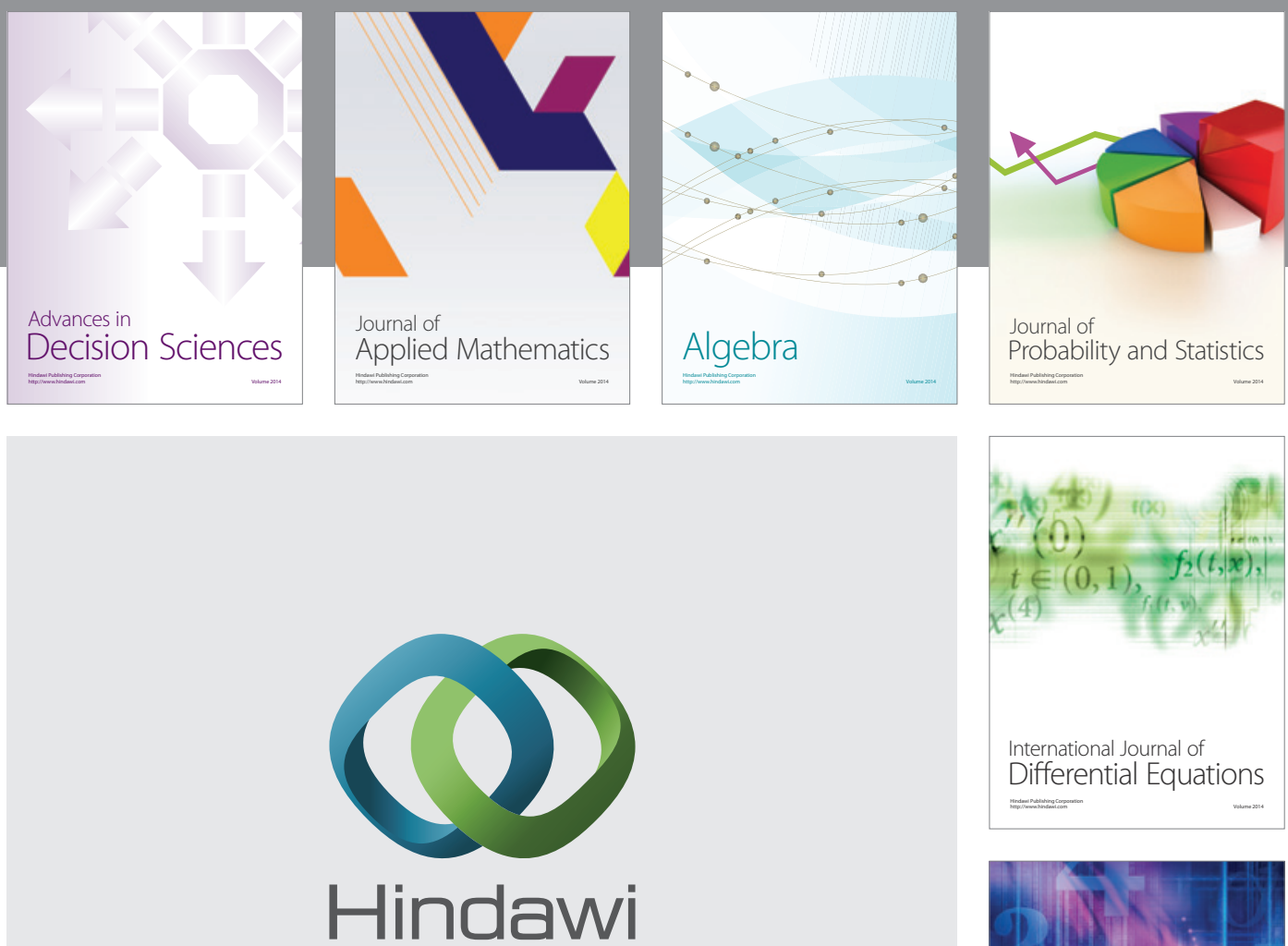

Submit your manuscripts at http://www.hindawi.com
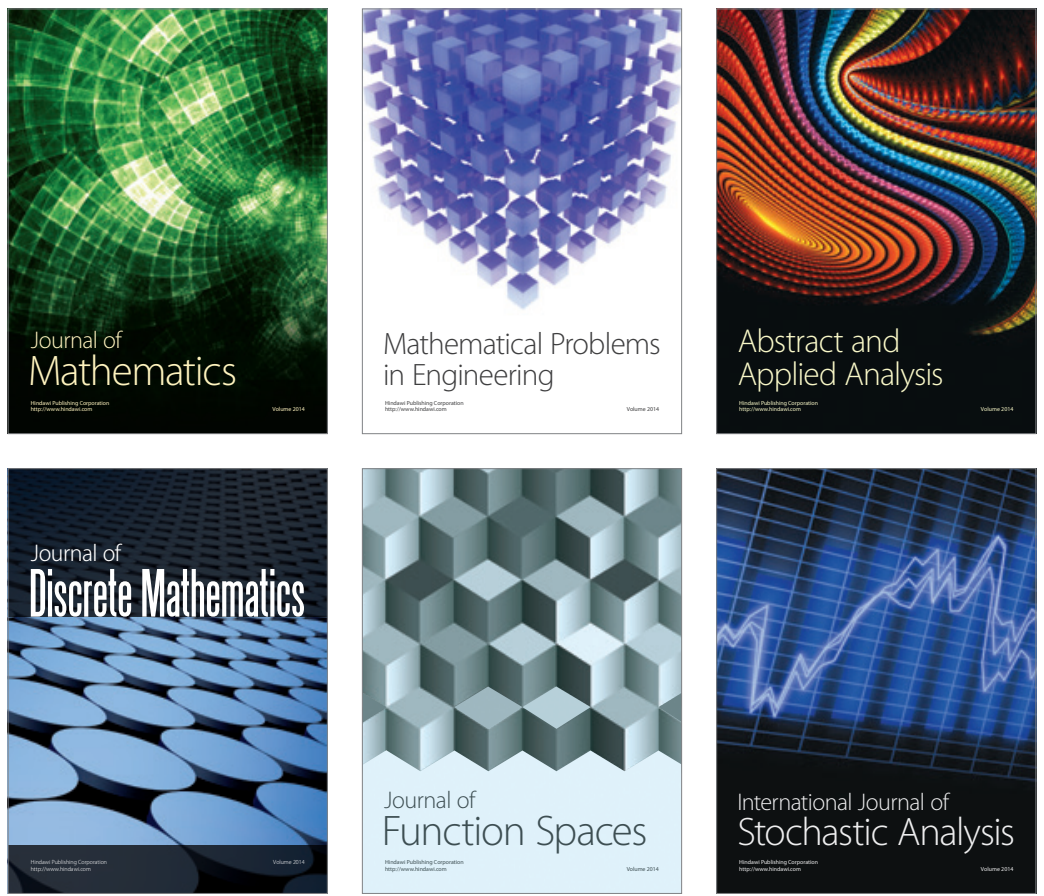

Journal of

Function Spaces

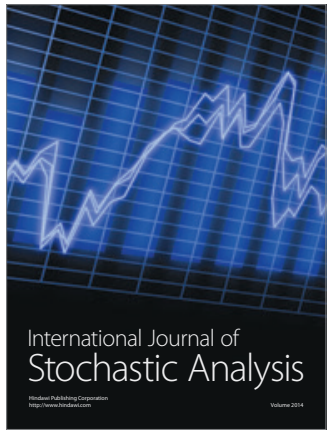

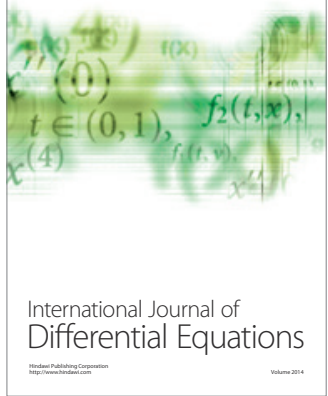
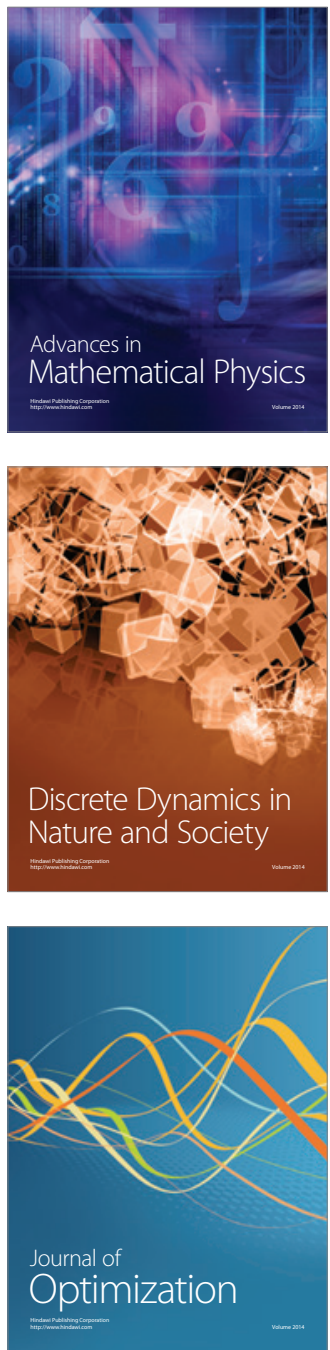